\title{
Current Measurements within the Electrospray Emitter
}

\author{
Boguslaw P. Pozniak and Richard B. Cole \\ Department of Chemistry, University of New Orleans, New Orleans, Louisiana, USA
}

\begin{abstract}
A movable disc-like wire probe electrode placed inside the electrospray (ES) capillary was used to measure currents flowing within the ES device for the first time. Currents were measured between the wire probe and the ES capillary. Current maps revealing measured current versus wire probe position were generated for a variety of solution conditions in the positive and negative ion modes and are compared to potential maps. The electrospray device was found to subsist on highly stable total currents; this current regulator aspect of the ES device showed remarkable resiliency regardless of the proportion of current produced at the wire probe electrode versus the ES capillary. However, kinks observed in the current and potential maps are attributed to adsorbed air participating in electrochemical reactions, and turbulence in solution flow in the region of the Taylor cone. From differential electrospray emitter potential (DEEP) maps, current maps, and cyclic voltammetry experiments performed at different wire probe locations, evidence is provided for separate regimes of current flow in the bulk solution and in the thin "skin" of highly conductive electrolyte constituting the outer surface (air interface) of the Taylor cone. Current maps reveal that current is drawn more evenly along the length of the ES capillary when solutions are highly conductive, in agreement with previous results for DEEP maps. In less conductive solutions, the area close to the capillary exit contributes more heavily to current production. Evidence that contaminant participation in electrochemical processes occurring within the electrospray device can be largely responsible for production of the excess charge in ES droplets is also provided. These investigations complement previous DEEP mapping studies to further elucidate the details of the electrochemical processes occurring within the electrospray device. (J Am Soc Mass Spectrom 2007, 18, 737-748) (C) 2007 American Society for Mass Spectrometry
\end{abstract}

$\mathrm{E}$ lectrospray (ES) [1-3] is a convenient tool to deliver ions from the liquid phase to the gas phase, so that they may subsequently be analyzed by mass spectrometry (MS). The inherent electrochemical nature of the ES device was first expounded by Paul Kebarle and coworkers [4, 5], but in many cases, as discussed in an excellent presentation of arguments by de la Mora, Van Berkel, Enke, and Fenn [6], experimentalists are often unaware of the electrochemical processes taking place during the operation of electrospray. In order to use electrospray prudently and efficiently, and to be able to remove chance elements, and extend its reach into new applications, a good grasp of the fundamental electrochemistry that underlies electrospray becomes necessary. Alongside the pursuit of practical applications of this atypical electrochemical cell comes an interest in explaining fundamentals of the electrochemical nature of electrospray [7].

The electrospray device-typically consisting of a capillary filled with liquid and a counter-electrode placed at some distance in front of it with a kilovolt

Published online January 25, 2007

Address reprint requests to Dr. Richard B. Cole, University of New Orleans, Department of Chemistry, 2000 Lakeshore Drive, New Orleans, LA 70148. E-mail: rcole@uno.edu potential difference between the two-was discovered to behave in a manner analogous to that of a controlled current electrochemical cell $[3,8,9]$. This characterization implicitly requires that electrochemical processes occur at each electrode to close the current loop. Despite the fact that in certain cases, droplet charging leading to electrospray can be accomplished without performing electrochemistry at electrical connectors [10], in a typical electrospray ion source, electrochemistry is invariably present.

In previous publications $[11,12]$ we presented measurements of potential distributions within metal electrospray capillaries using a method that we call "differential electrospray emitter potential" (DEEP) mapping. Construction of these potential maps, along with investigations of the factors that influence their shapes, were conducted in both the positive [11] and negative [12] ion modes. The present paper complements our previous efforts in potential mapping by adding investigations of current distributions. Although current density and interfacial potential are theoretically dependent $[13,14]$, exact equations relating these two parameters require a detailed knowledge of every step of molecular transformation. In typical ES-MS studies involving large organic molecules, this information is not readily obtainable. Moreover, pitfalls encountered in potential 
measurements are different from those pertaining to current measurements, which renders the two more complementary than redundant. Practical difficulties and contributions to error in making potential measurements and creating DEEP maps were previously discussed in detail [11].

\section{Theoretical Introduction}

To aid in the interpretation of current map data, and to contrast this information with that obtained from potential maps, it is practical to distinguish between primary, secondary, and tertiary current distributions [15-17]. A primary current distribution takes into consideration only the potentials of the electrodes; the fashion in which current is distributed is determined only by the relative resistances of possible current paths. This is an idealized starting point-in practice it may describe current flow between two ideally nonpolarizable electrodes. A secondary current distribution includes resistance at the electrode surface. This resistance comes from the barrier to electron transfer at the electrode surface and, unlike ohmic resistance, it is a nonlinear function of potential. A secondary current distribution is applicable when current densities at all electrode localities are below the limiting current. The limiting current is the maximum current obtainable under local mass transport conditions; it is smaller than the current predicted by the current-potential relationship. A tertiary current distribution includes local mass transport conditions. If confronted only by a potential map (that is, no other information is available), one is able to gauge only the primary distribution of current. With some additional knowledge of the electrochemistry taking place, one can get an indication of the secondary distribution, although to deduce the tertiary distribution, one must also have knowledge of mass transport conditions. Of course, the tertiary current distribution corresponds most closely to reality, but, in a comparison of current and potential maps, if it is assessed that the conditions approximate a primary or secondary current distribution, this allows one to draw certain conclusions regarding the nature of the electrochemistry, the mass transport conditions, and so forth. A complete inventory of currents in the electrolyte is theoretically attainable only by solving the Laplace equation for the bulk solution with proper boundary conditions at the electrodes and nonactive elements of the electrochemical cell. In most instances only numerical solutions are possible and, even then, considerable effort is required [18, 19].

Electrical potential, by definition, is never an absolute value; it must always be defined with respect to a reference point $[17,20]$. For every electrode immersed in an electrolyte there exists a potential difference between the electrode and the electrolyte. This difference is called the interfacial potential, which cannot be measured directly. Interfacial potential should not be confused with the potential applied to the metal elec- trode from the power source. In an electrochemical cell, when current flows between two electrodes, different locales of the same electrode will have unequal interfacial potentials, and consequently different current densities, despite having the same potential within the metal. Differences in local interfacial potentials occur because the current between electrodes may flow through the electrolyte by a large variety of paths. The total current, representing the sum of all individual current paths, will distribute itself through these paths in such a way that overall dissipation (loss) of energy is minimized.

A potential measurement between two electrodes is, in fact, a measurement of the difference of two interfacial potentials, with each of the latter already constituting a difference between the potential of the respective metal and the local electrolyte. To reliably measure a potential difference, the set of electrodes must include a reference electrode, whose interfacial potential is practically constant, so that the measurement reflects changes of interfacial potential only at the electrode of interest. One of the necessary characteristics of a reference electrode is fast kinetics of the electrode reaction. This guarantees that when current passes, the potential drop between the metal and the electrolyte constituting the reference is so small that it can be ignored. In other words, a good reference electrode should pose no resistance to the flow of current.

In an electrospray device, it is not customary to place a reversible couple of reactants at the electrode surface, as is normally done in electrode referencing. An unfortunate outcome of using a pseudoreference electrode within the ES device is that the obtained interfacial potential may be determined by an accidental set of chemicals that happens to be there, such as contaminants. Then, the measured potential will reflect not only changes in the interfacial potential of the electrode of interest, but also changes in the interfacial potential of the pseudoreference. Even small quantities of impurities are often able to compete with reactions of intentionally added solution constituents occurring at the electrode surface, resulting in a drift of the potential of the electrode chosen as the reference, thereby subjecting the measured potential to substantial errors.

By comparison, current measurements are relatively free from the above-described undesirable electrode behavior because, to generate faradaic current, real work must be done (that is, transport of electrochemically active species to and from the electrode and transfer of electrons through the interface must occur). Small amounts of contaminants are not capable of providing significant currents, which often means that current measurements are more indicative of the major electrochemical processes compared to potential measurements. On the other hand, current measurements are subject to limitations pertaining to the mass transport situation in the electrolyte, to activation overpotential, and to concentration overpotential. The experimental (true, tertiary) current distribution will depend on 
factors that do not influence measured potential, so the set of results of current measurements presented here complements the picture described by previous potential measurements in the ES capillary [11, 12].

\section{Experimental}

A schematic diagram of the instrument used in this investigation is presented in Figure 1. Details of this setup were presented previously [11, 12, 21]. In brief, experiments were done with a custom-built standard electrospray device, connected to an electrochemical work station (Model 660A, CH Instruments, Austin, TX). Voltage to the counter-electrode was set by a high-voltage power supply (Glassman, High Bridge, NJ). A 99.95\% platinum ES capillary was purchased from Goodfellow Cambridge Ltd. (Huntington, UK), with a $99.9 \%$ platinum wire used for the central probe electrode purchased from Alfa Aesar (Ward Hill, MA).

Chemicals used in these investigations came from the following sources: methanol and acetonitrile (both HPLC-grade, EMD Chemicals, Gibbstown, NJ); chloro- form (chromatography-grade, J. T. Baker Inc., Phillipsburg, NJ); potassium chloride (Mallinckrodt, St. Louis, $\mathrm{MO}) ; 1,4$ benzoquinone, lithium trifluoromethylsulfonate $\left(\mathrm{LiCF}_{3} \mathrm{SO}_{3}\right)$, and rubrene $\left(\mathrm{C}_{42} \mathrm{H}_{28}\right)$ (Sigma-Aldrich, St. Louis MO). The above chemicals were used as supplied without further purification. Distilled, deionized water was obtained using a Milli-Q water system (Millipore, Billerica, MA).

The (wire) probe electrode (Figure 1) consists of a platinum wire shielded and sealed within a fused silica capillary such that only the tip of the wire, in the form of a small disc, is in contact with the electrolyte. On the back end, the wire in its shield passes through a "cross" connector to the outside environment, where it is attached to a mechanical stepping motor. The entire shielded wire moves (slides) inside the surrounding electrospray capillary; the exposed tip of the wire (disc electrode) may be positioned at different places with respect to the ES capillary exit. The coordinate system is chosen such that when the end of the wire is flush with the ES capillary exit, it is considered to be positioned at the "zero" point; positive values of " $x$ " indicate that the

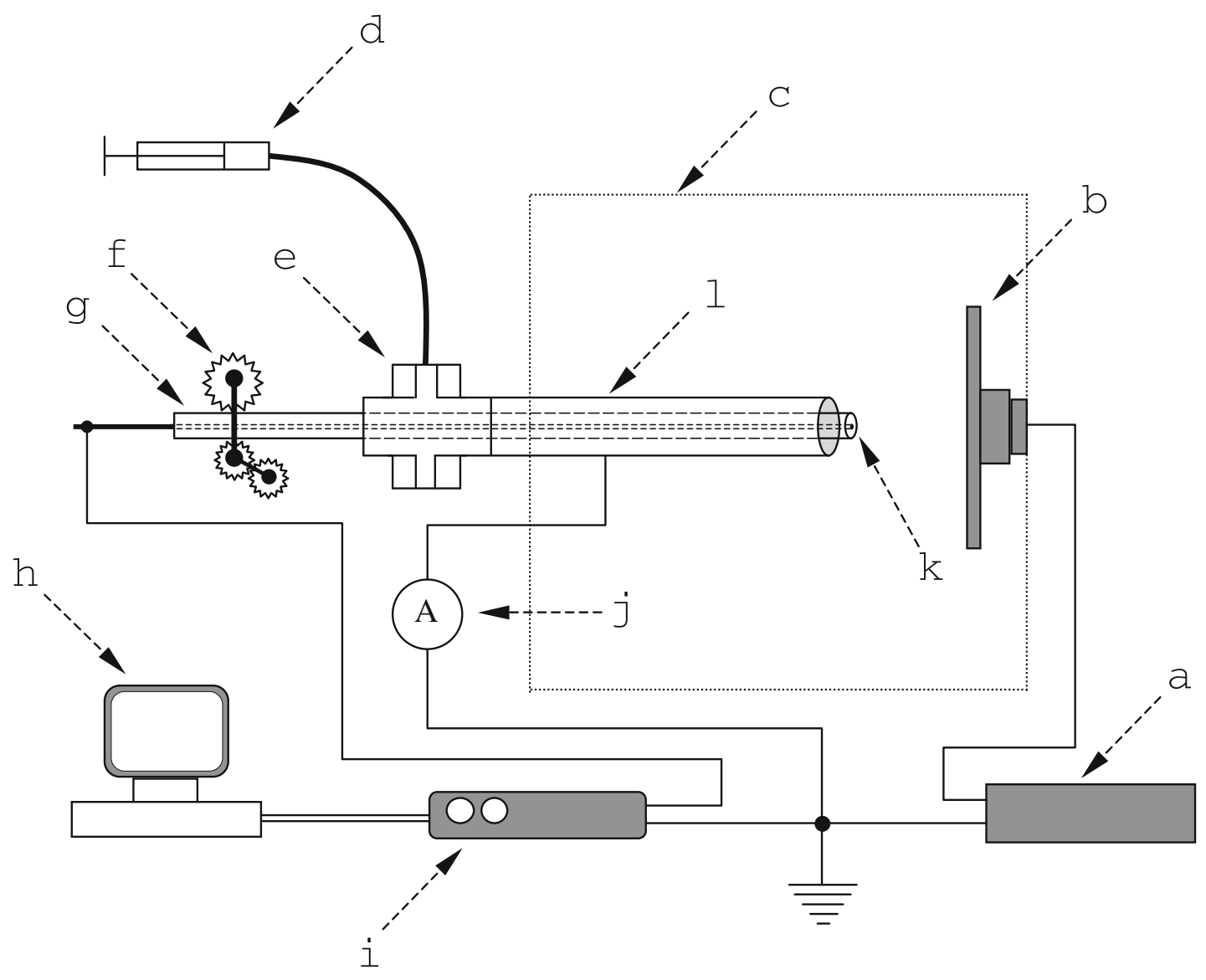

Figure 1. Schematic diagram of the experimental device used to make current and potential measurements: (a) high-voltage power supply; (b) brass plate serving as ES counter-electrode; (c) Faraday cage; (d) syringe pump; (e) cross union; (f) mechanical device for positioning wire probe electrode assembly; (g) fused silica capillary; (h) computer workstation; (i) potentiostat; (j) ammeter; (k) Pt wire probe electrode assembly sealed in fused silica exposing a disc-like surface; (l) Pt ES capillary. 
wire is sticking out of the capillary (entering the volume of the Taylor cone), whereas negative values of " $x$ " mean that the wire end is retracted inside the ES capillary. The wire can be moved either manually, and its position read using a calibrated micropositioner, or mechanically, with its position determined from the relationship between velocity of travel and time.

Hardware parameters: platinum electrospray capillary internal diameter $500 \mu \mathrm{m}$, outside diameter $600 \mu \mathrm{m}$, cylindrical wire electrode $(0.127 \mathrm{~mm}$ diameter $)$ with fused silica capillary shield (inside diameter $180 \mu \mathrm{m}$, outside diameter $220 \mu \mathrm{m}$ ), electrolyte flow rate $200 \mu \mathrm{L} / \mathrm{h}$ delivered by syringe pump (Orion 341B, Sage Instruments, Boston, MA). This makes the linear velocity of the liquid inside the ES capillary $0.28 \mathrm{~mm} / \mathrm{s}$ in places where the wire electrode is absent and 0.48 $\mathrm{mm} / \mathrm{s}$ in places where it is present.

In open-circuit potential measurements, the wire probe electrode is connected to the reference electrode lead of the workstation, whereas the working electrode lead is connected to the ground of the highvoltage power supply. The same ground is connected to the ES capillary through a battery-powered ammeter. Because the ES capillary is grounded, in order to to work in the positive ion mode, the opposing brass plate counter-electrode is held at high negative potential; for negative ion mode operation, the counter-electrode is at high positive potential with respect to ground. Because the work station leads are reversed relative to conventional operation, a change of sign is made in all plots to convey proper polarities. Open-circuit potential measurements with small electrodes and highly resistive electrolytes require extremely high input impedances of electrochemical equipment. When the instrument draws even a small current, it may greatly distort the measured potential. Sources and relative magnitudes of errors in open-circuit potential measurements made within the electrospray capillary were discussed in detail in a previous publication [11].

For current measurements, the electrochemical work station working electrode lead was attached to the wire probe electrode, and the counter- and referenceelectrode leads were grounded. The potential difference between the wire probe (working) and ES capillary (reference) was set at zero (that is, they are at the same potential). With such settings, the instrument readout indicates current flowing to (or from) the working electrode. An ammeter is still connected in series to the ES capillary, so the current to the ES capillary is also measured, and the sum of currents to the wire probe and ES capillary can be calculated afterward. An experiment can also be done whereby a certain potential difference (positive or negative) is set between the wire probe and ES capillary electrodes. Following the standard convention, negative, or oxidative current is termed anodic, positive, or reductive current is cathodic.

The brass plate counter-electrode is placed at a distance of $8 \mathrm{~mm}$ from the ES capillary tip. A typical
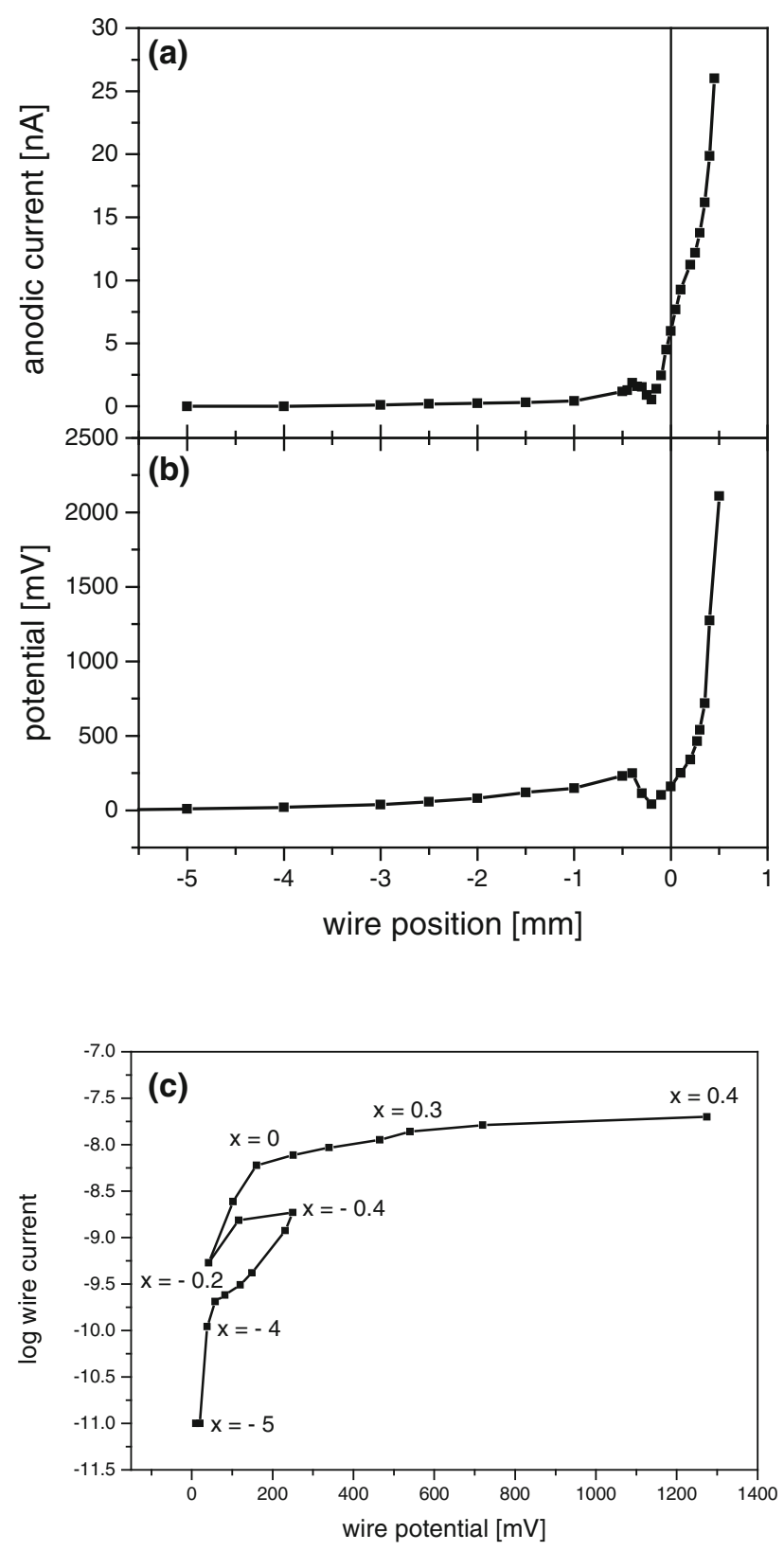

Figure 2. Positive ion mode ES operation in an ambient air atmosphere: (a) current; (b) potential maps, both obtained in methanol:water 50:50; (c) Tafel plot of data from (a) and (b).

high-voltage setting was $3300 \mathrm{~V}$. The exact value varied slightly depending on the solvent used. All measurements were performed under the protective atmosphere of nitrogen gas with one exception as noted in the text. All experiments were done in the single-jet mode of electrospray, according to the Clopeau classification [22].

\section{Results and Discussion}

\section{Methanol}

Figure 2 shows potential and current maps for a 50:50 (vol/vol) methanol:water mixture with electrospray 
operating in the positive ion mode. In this mode, oxidation reactions are predominant. The potential of the wire electrode (Figure $2 b$ ) is positive relative to the ES capillary at all positions. Gradients of potential are the largest where the wire probe enters the Taylor cone, as indicated by the largest slope of the potential map. In moving away from the Taylor cone (from the extreme right to the left in Figure $2 b$ ) the steep decline of the potential map is momentarily reversed and, at slightly negative values of $x$, the potential starts to increase, resulting in a kind of "dip" in the potential map.

Figure 2a shows a current map, that is, the oxidative current at the wire probe as a function of its position. Total current is now divided between the wire probe and the ES capillary. The total current (that is, the sum of currents at the wire probe and the ES capillary) remains unchanged (about $31 \mathrm{nA}$ in this experiment) regardless of the position of the wire probe. This conservation of total current, despite its division into two electrodes, is evidence of the fact that the ES source operates as a controlled-current electrochemical cell $[4,8,9]$. The current "regulator" can be viewed as the place where the filament of liquid is formed at the apex of the Taylor cone. Experimental and theoretical aspects of this issue were previously discussed in the works of Kebarle, Van Berkel, and de la Mora [3, 4, 23-25]. In theory, there are many parameters that can influence the total ES current; among them are the strength of the electric field and liquid properties such as: conductivity, dielectric constant, surface tension, viscosity, and flow rate. In practice, however, for a given solvent, only a small change of current is tolerable before instability disrupts ES operation. The only parameter that can be readily varied over a rather wide range (roughly two orders of magnitude) while maintaining spray stability is conductivity; the flow rate can be varied at most by a factor of about 4 , and the strength of the electric field (high voltage) by a factor of only about 1.2. Unlike the situation for spraying liquid metals, in aqueous electrolytes the shape, size, and vortex angle of the Taylor cone are not constant [22, 26-28]. A change in one parameter, such as the electric field strength, leads to a change in Taylor cone shape, including the position where the filament is formed. This, in turn, alters the distribution of current in the ES capillary, thus distorting the shape of the current map. For the above reasons, our initial study of current maps within the ES emitter investigates single solvents with different conductivities stemming from different concentrations of supporting electrolyte.

By necessity, potential measurements were done using a two-electrode system, in which we have assigned one electrode as the indicating (working) electrode and the other as the (imperfect, polarizable) reference. In open-circuit potential measurements, the working electrode potential is quite sensitive to even small amounts of electroactive species because its potential is determined not by the most abundant species, but by the most electroactive-that is, those that exchange electrons with the electrode at the fastest rate.
As a consequence, chemicals produced upstream to the wire probe may change its potential, because the actual measured potential is the sum of half potentials of the electrode reactions in the cell and the $\mathrm{iR}$ drop between them (primary current distribution). This problem is minimized in current mapping. To produce current, a substance must be present in quantities that correspond to Faraday's law, so small amounts of upstreamproduced chemicals will not alter the picture. Nonetheless, if generated upstream chemicals or contaminants are present in substantive quantities, they may become the principal electroactive species if liquid flow conditions and mass transport to the electrode surface are favorable.

The current map (Figure 2a) shows the same kind of "dip" as the potential map (Figure 2b). The positional correspondence of these two dips indicates that this irregularity is not an artifact. Figure 2c shows a plot of wire probe current versus wire probe potential in the manner of a Tafel plot. This plot shows that for certain potential values, more than one amount of current can be produced, depending on the position of the wire probe inside the ES capillary. This violates the principle of a one-to-one correspondence between current and potential of the electrode and leads to the conclusion that, at different places within the ES capillary, different electrode reactions are occurring.

The most likely explanation for this behavior is that locally formed electrochemical "cells" have different electrolyte compositions. We attribute the local change in solution composition to the presence of ambient gas, that is, oxygen-containing air. Air is adsorbed through the "skin" of the Taylor cone and it then becomes involved in electrochemical processes. Positions far upstream in the ES capillary are not influenced by the gas adsorbed at the ES capillary exit, so the nature of the electrochemistry occurring there is different. We recently showed [29] that the composition of ambient gas can have a substantial influence on the potential map; consequently, it also has an influence on current maps. This "oxygen effect" is amplified by turbulent liquid flow inside the Taylor cone. Figure $2 \mathrm{a}$ and $\mathrm{b}$ reveal that this turbulent reactor region is more or less confined to the Taylor cone and the first $0.5 \mathrm{~mm}$ length into the ES capillary. At points deeper than about $0.5 \mathrm{~mm}$, flow is more orderly in the capillary and oxygen does not appear to be transported that far upstream.

When a similar experiment is conducted in a protective atmosphere of nitrogen, the DEEP map is monotonically growing in the positive direction (Figure $3 b$ ). The current map (Figure 3a) and the plot of current versus wire probe electrode potential (Figure 3c) are also monotonic. The curve in Figure $3 c$ has the features that one would expect of a typical electrolysis. There is practically no current below a certain potential (about $30 \mathrm{mV}$ ), then current rises sharply, and finally levels off above $800 \mathrm{mV}$. The smoothness of the latter plot indicates a consistency in measurement, which is reassuring in our case, where we lack a true reference electrode. In 

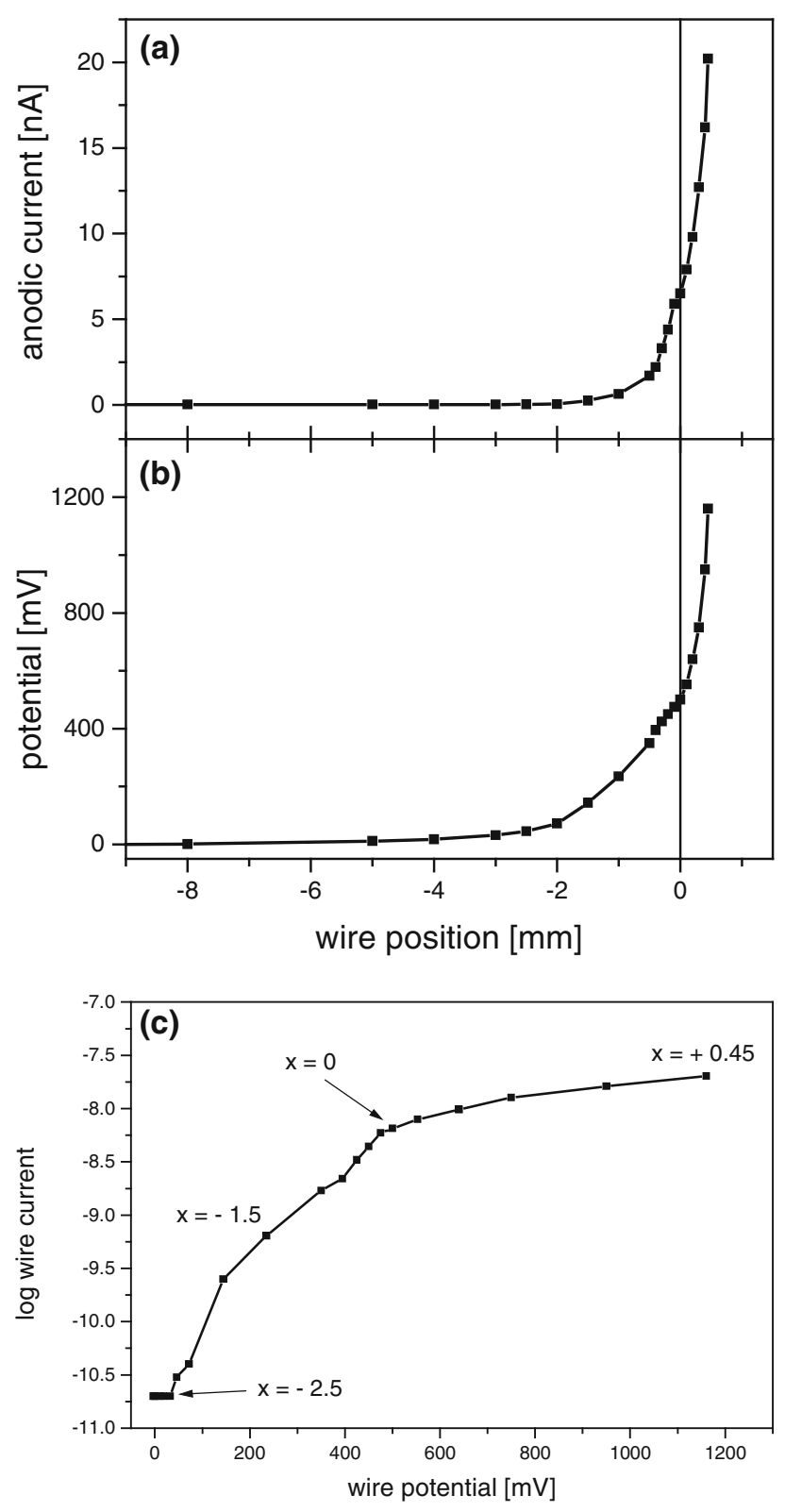

Figure 3. (a) Current and (b) potential maps obtained in the positive ion mode from $100 \%$ methanol with ES operating in a nitrogen atmosphere. (c) Tafel plot of data from (a) and (b).

contrasting Figure $3 \mathrm{c}$ with Figure 2c, the absence of a "kink" in the former is not an indication of the absence of turbulence in the Taylor cone, but rather, a reflection of the fact that now an altered chemical environment is no longer created by entry of reactive gas at the Taylor cone.

Turbulent flow of liquid inside the Taylor cone is readily observable through a microscope when fine alumina powder is added to the spraying liquid. Clearly, there is a backflow along the ES capillary axis that drives electrochemical reaction products back into the capillary. Turbulent behavior at the ES capillary exit was documented in a series of publications by Hayati and coworkers and the existence of back current in the
Taylor cone was photographed [26-28]. The basis for such turbulence was also examined from first principles in fundamental hydrodynamic studies [33]. It was suggested by de la Mora [23] that backflow may be specific only to solutions of sufficiently high conductivity. He also advanced the idea that a larger portion of the total current is being conducted through the "skin" of the Taylor cone- a charged liquid-gas interfacial regionthan through the bulk electrolyte. The shearing stress caused by the fast moving charge carriers of the skin was suggested as the underlying cause of the liquid backflow along the capillary axis. Turbulence will inevitably occur as backflow collides with the pressuredriven forward-flowing liquid.

\section{Acetonitrile and Influence of Solution Conductivity on Current Distribution}

Current maps for three different solutions of acetonitrile are shown in Figure 4. This experiment is done in
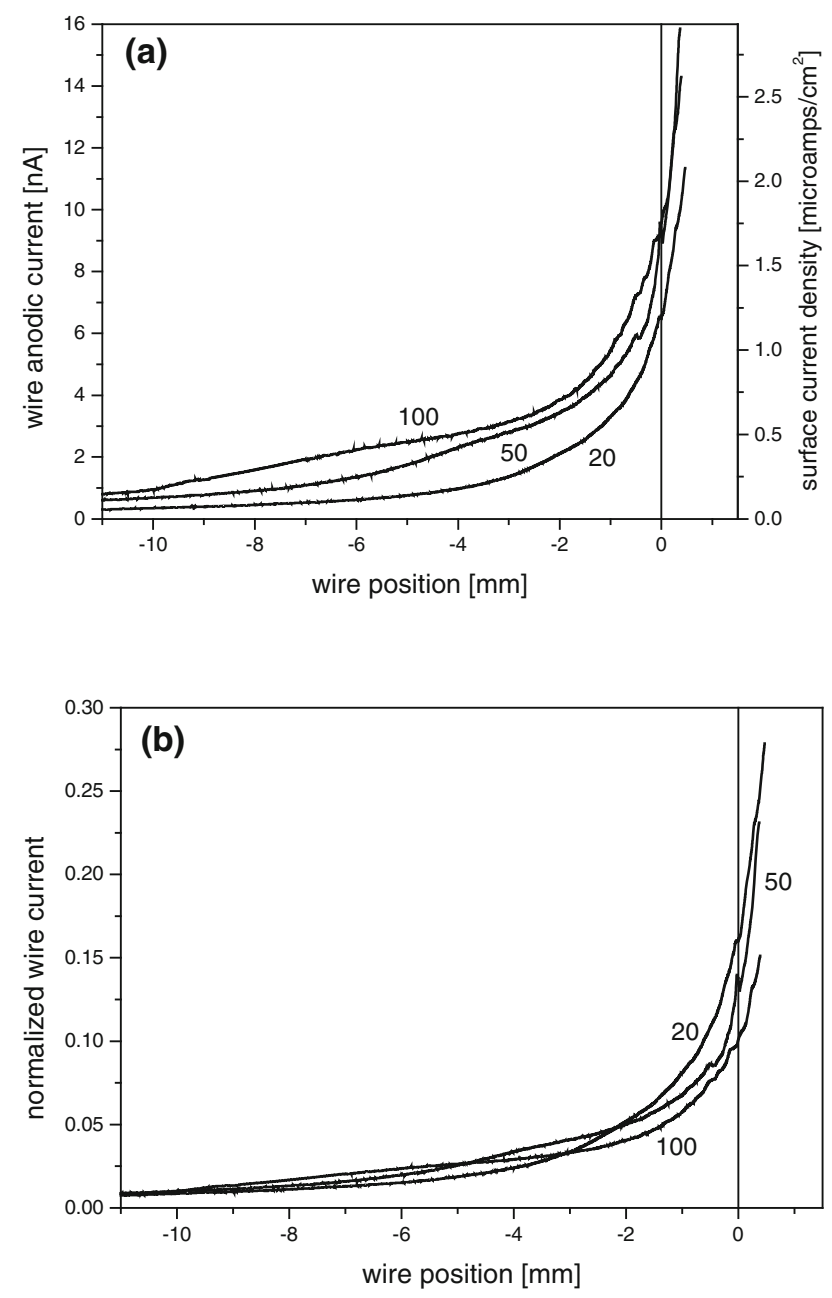

Figure 4. Positive ion mode current maps obtained from $100 \%$ acetonitrile with lithium trifluoromethylsulfonate supporting electrolyte at 20, 50, and $100 \mu \mathrm{M}$. (a) Measured current versus wire probe position; (b) same data normalized to total electrospray current (values in the text). 
the positive ion mode, where the dominant process at the ES capillary is anodic oxidation. Total electrospray current for $20 \mu \mathrm{M}$ solution is $40.1 \mathrm{nA}$, for $50 \mu \mathrm{M}$ it is $68.6 \mathrm{nA}$, and for $100 \mu \mathrm{M}$ it is $94.6 \mathrm{nA}$. When plotted versus supporting electrolyte concentration, the total current values can be fitted with a square-root function,

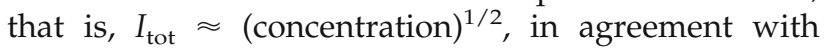
previous findings $[7,8,23,34]$. Figure 4 a shows that for the most concentrated solution $(100 \mu \mathrm{M})$, the wire probe current is the highest at all points of $x$. Even for points quite deep into the ES capillary, there is still a significant current for the $100 \mu \mathrm{M}$ solution, whereas the current approaches zero at positions much closer to the exit for the less concentrated solutions. However, at wire probe positions close to $x=0$, the highest concentration curve does not increase slope as rapidly as do the others. The issue is more clearly seen in Figure $4 \mathrm{~b}$ where the current map is plotted as normalized current, that is, all values on a given current map are divided by the total current. The conclusion is that, for a more conductive solvent, the distribution of current is more even along the capillary length, whereas for a less conductive solvent, the current production occurs principally in the region close to the capillary exit. To produce a given current when solution resistance is high, an additional stretch of the current path through the bulk electrolyte causes a relatively large potential drop. For lower solution resistances, there is a gain in distributing current over a larger surface area (reaching further into the ES capillary) because the local current densities gradients near the ES capillary exit are less steep and the interface potentials are dropping more slowly as the capillary is entered. The trade-off between increasing the length of electrolyte to traverse and decreasing the interfacial potential at greater distances within the ES capillary dictates the viable current paths and determines the shapes of current (and potential) maps. This picture reinforces the conclusions drawn from potential maps [11] and is consistent with previous depictions of current distributions in electrochemical cells [24, 30, 31].

The right axis of Figure 4 a shows the current densities on the inner walls of the ES capillary as calculated by integration of the current map from minus infinity to $x=0$, and then accounting for the total current and the known capillary inner surface area. It should be noted that the wire probe electrode itself generates a nonnegligible amount of current, which, in turn, leads to changes in current distributions. The most rigorous treatment would include a full deconvolution of data to compensate for this effect. Nevertheless, because the current produced by the wire probe is only a fraction of the total current (15\% at most), the right axis numbers are still reasonably informative.

Integrating from minus infinity to $x=0$, of course, ignores currents measurable inside the Taylor cone. These additional current paths must terminate at the ES capillary and their existence implies that the current (or potential) registered by the wire probe at a given position of $x$ does not correspond directly to the current (or potential) at the ES capillary walls at the same $x$-value. Rather, there is a shift whereby the wire probe currents (potentials) measured at positive values correspond to currents (potentials) at points within the ES capillary. Further complicating matters is evidence that current is not exclusively produced at the inner walls of the ES capillary. De la Mora [23] clearly distinguished between the current conducted by the Taylor cone "skin" and the bulk electrolyte inside. The Taylor cone skin may conduct current directly through the capillary front surface. The wire probe is capable of measuring current only through the bulk electrolyte. In fact, when the wire probe touches the skin of the Taylor cone, the current jumps rapidly and erratically, similar to what was observed for potential in open-circuit measurements.

Current densities in Figure $4 \mathrm{a}$, for $x=0.0 \mathrm{~mm}$, range from about 1.2 to $1.8 \mu \mathrm{A} / \mathrm{cm}^{2}$. One can calculate that a substance present at $1 \mu \mathrm{M}$, exchanging two electrons in the electrolysis and having a $100 \%$ conversion rate, is capable of providing the entire current. In the case of commercially available acetonitrile, this substance can be water, present as an impurity. Acetonitrile is very difficult to oxidize [35] and its oxidation on a platinum surface is a complex process. The oxidation potential of water $(1.35 \mathrm{~V})$ is significantly lower than that of acetonitrile $(2.3 \mathrm{~V})$ and it is further lowered in the presence of oxygen. Because even "dried" acetonitrile can contain millimolar quantities of water, a conversion rate of far less than $100 \%$ would be required to provide all of the measured current by oxidation of water. Moreover, to achieve current densities of about $2 \mu \mathrm{A} / \mathrm{cm}^{2}$ from contaminant water oxidation, only modest overpotentials (say about $0.2 \mathrm{~V}$ ) are required. This low overpotential is a minor portion of the previously measured potentials for acetonitrile $(>1 \mathrm{~V}$ at $x=0)$ [11] and it corroborates our postulation that most of this $1 \mathrm{~V}$ is created by $\mathrm{iR}$ drop in the solution with an additional contribution from concentration overpotential.

Another issue related to current distribution is the ES capillary geometry. The front end surface (closest to the counter-electrode) of the platinum annulus has a nonzero wall thickness such that it is in direct contact with the skin of the Taylor cone. For the ES capillary used, if the front surface were delivering the current density measured for $x=0 \mathrm{~mm}$, it would be responsible for only $2.5 \%$ of the total current production. However, as previously suggested, the electrical conductivity of the Taylor cone skin is likely to be significantly greater than that of the bulk and thus, the front surface could be producing a far greater portion of the current. Even at the most positive $x$ position, the fact that the wire probe delivers only $20-30 \%$ of the total current supports the notion that the Taylor cone skin is responsible for passing the majority of the current via the front capillary wall. 


\section{Negative Ion Mode}

Figure 5a shows current maps for methanol with $\mathrm{KCl}$ as supporting electrolyte. The current in the negative ion mode of operation is positive (cathodic) and the predominant electrode process is reduction. The total current is $26 \mathrm{nA}$ for the $10 \mu \mathrm{M}$ solution, $56 \mathrm{nA}$ for $50 \mu \mathrm{M}$, $79 \mathrm{nA}$ for $100 \mu \mathrm{M}$, and $156 \mathrm{nA}$ for $500 \mu \mathrm{M}$. When total current is plotted against concentration (not shown), only the first three points can be reasonably fit to a square-root function. If the fourth point $(500 \mu \mathrm{M})$ is included, a lower exponent (such as $3 / 7=0.428$ ) provides a better fit. The exponent $3 / 7$ happens to be that predicted by Hendrix theory [5], although various authors have evaluated this exponent differently $[7,8$, 23, 34].

Current maps in Figure 5a mirror those obtained in the positive ion mode. The solution with the highest concentration of supporting electrolyte provides the highest current in all places. In Figure 5b, the current maps are normalized. It is seen that the solution with the highest concentration of supporting electrolyte dis-
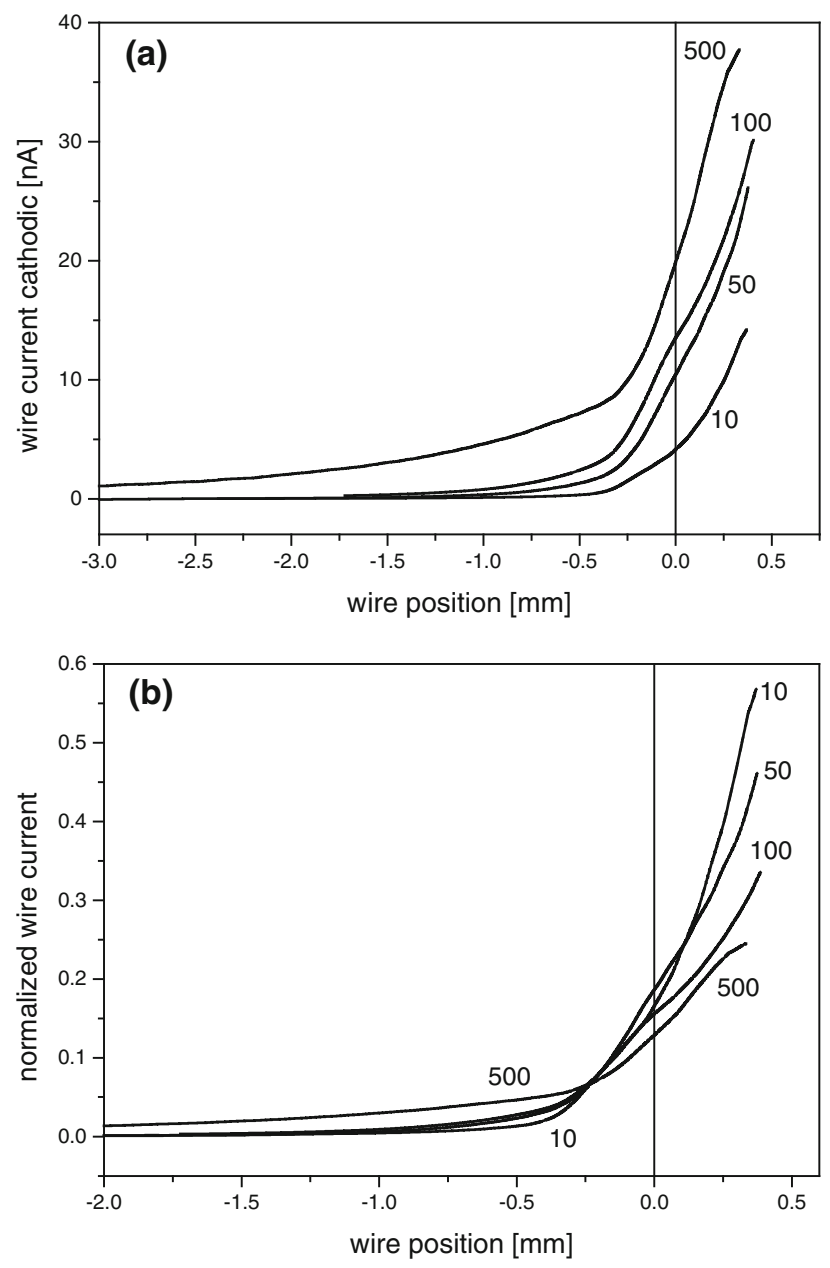

Figure 5. Negative ion mode current map obtained from $100 \%$ methanol with potassium chloride supporting electrolyte, 10, 50, 100 , and $500 \mu \mathrm{M}$. (a) Measured current versus wire probe position; (b) same data normalized to total current (values in the text). tributes the total current more evenly along the capillary. The opposite is observed for the least concentrated solution-that is, the front part of the ES capillary produces most of the current. The current maps in Figure 5 appear to consist of two different regimes merged together. The division point is at about $x=$ $-0.3 \mathrm{~mm}$, which is slightly more than the internal diameter of the capillary. Likely at this point, the nearly laminar flow conditions inside the capillary meet with the turbulent reactor zone of the Taylor cone.

If we consider that there are two independent electrochemical reactions occurring, with neither of them being diffusionally limited, the theoretically predicted current map would be a sum of the two individual exponential curves that, at a glance, would also look like an exponential function. A sudden change of slope would not be anticipated. The abrupt slope shift observed in Figure $5^{\circ}$ suggests ${ }^{\circ}$ that ${ }^{\circ}$ the ${ }^{\circ}$ reaction having ${ }^{\circ}$ the lower $E^{\circ}$ may be reaching its diffusional limit near $x=$ $-0.3 \mathrm{~mm}$. When a reaction is diffusionally limited, the current is less a function of interface potential than of mass transport conditions. Trace organic impurities are likely to be present in micromolar quantities in commercial solvents, whereas water and dissolved gases (notably oxygen) can be present at up to millimolar levels. At a certain point in the ES capillary, after reaching a threshold interfacial potential, the major reaction starts to dominate; upstream from that point, current production is driven mostly by impurities. An additional underlying cause for the consistent bends in the ${ }^{\circ}$ Figure $^{\circ} 5^{\circ}$ maps $^{\circ}$ may $^{\circ} \mathrm{be}^{\circ} \mathrm{a}^{\circ}$ hydrodynamic ${ }^{\circ}$ effect. ${ }^{\circ}$ The reaction(s) are diffusionally limited, although as the turbulent Taylor cone region is entered (starting at about $-0.3 \mathrm{~mm}$ as previously noted), better mixing is occurring. As a consequence, the thickness of the diffusional layer may be reduced with the effect of increasing diffusionally limited currents.

\section{Easily Oxidizable Species}

Rubrene is a polycyclic aromatic hydrocarbon that undergoes oxidation at low potentials $\left(E_{1 / 2}=0.77 \mathrm{~V}\right.$ versus SCE); a DEEP map for rubrene was presented in a previous publication 911$]$. In Figure $\%,{ }^{\circ}{ }^{\circ}$ current ${ }^{\circ}$ map is presented, with the current versus potential plot shown as an inset. The presence of two regimes of current production is even more pronounced than in the previous example. However, the potential map shows no

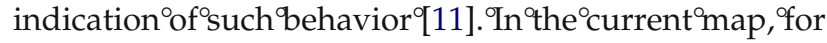
locations deep in the capillary where the interfacial potential is relatively low, oxidation of unknown contaminants with oxidation potentials even lower than that of rubrene is likely to be occurring. Only after reaching a certain "firing" potential, at about $x=$ $-0.5 \mathrm{~mm}$, does oxidation of rubrene start in earnest. At values more positive than $x=-0.5 \mathrm{~mm}$, current increases linearly with potential over the remaining measurable range (see inset). This linearity implies: (1) that only small overpotentials are present and (2) that the 


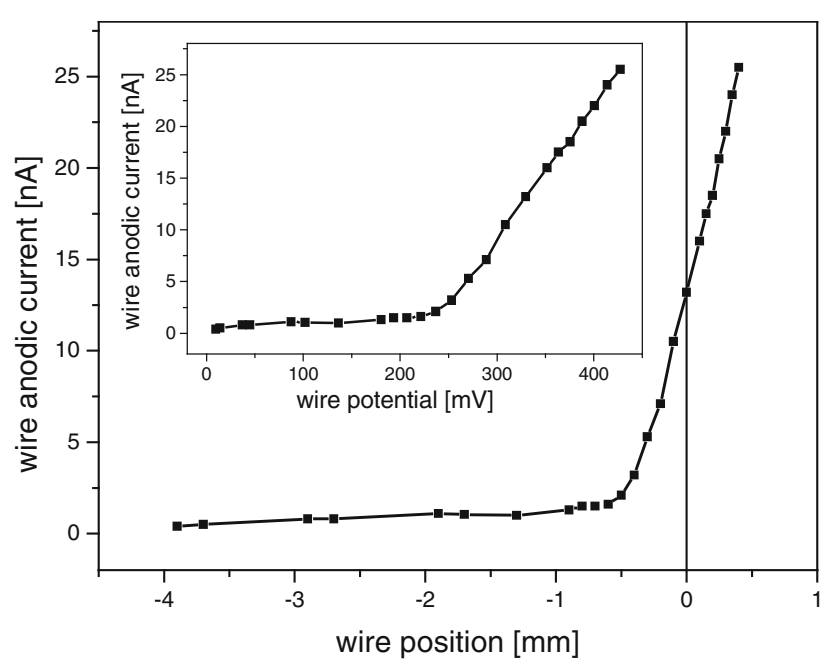

Figure 6. Positive ion mode current map from rubrene in $100 \%$ acetonitrile with $100 \mu \mathrm{M}$ lithium trifluoromethylsulfonate supporting electrolyte; total anodic current is $115 \mathrm{nA}$. Inset: Tafel plot using same data.

electrode reaction is not mass transport limited in this turbulent region. Integration of the current map from minus infinity to $x=0$, with accounting for flow rate and rubrene concentration $\left(10^{-5} \mathrm{M}\right)$, indicates that roughly $50 \%$ of the total electrospray current is produced by contaminants in the low-potential regime; oxidation of rubrene provides the remainder. Thus, even for easily oxidizable species, achieving good conversion rates may be limited by the amount of contaminants in the electrospraying solution, a conclusion previously ${ }^{\circ}$ formulated ${ }^{\circ} \mathrm{by}^{\circ}{ }^{\circ} \operatorname{an}^{\circ}{ }^{\circ} \operatorname{Berkel}^{\circ}[9]$.

\section{Wire Probe Electrode as an Electrode of Chosen Polarity}

Switching of current from the wire probe to the ES capillary. Use of a metal spray capillary is not essential to successful electrospray operation. It is well known that the electrospray current may be generated at a point of metal-solution contact that is far upstream from the spray tip and counter-electrode. Shown in Figure ${ }^{\circ} 7^{\circ}$ are ${ }^{\circ}$ the ${ }^{\circ}$ results ${ }^{\circ}$ of ${ }^{\circ}$ experiments ${ }^{\circ}$ where ${ }^{\circ}$ current production is switched from the ES capillary to the wire probe using three different electrolyte concentrations. The position of the wire was deliberately chosen to be fixed deep inside the ES capillary, that is, $x=-7.5 \mathrm{~mm}$, where it produces minimal current. Upon disconnection of the lead to the ES capillary (marked as ES capillary "off"), the site of current production shifts from the ES capillary walls to the surface of the wire probe, but no change of Taylor cone shape is perceptible. Clearly, the electrical capacitance of the system is sufficiently high to absorb the alteration in current production. With the ES capillary disconnected, the total current is produced at the wire probe. Because of its small surface area (roughly $1 / 7$ that of the front exposed surface of the ES capillary), the current density on the wire probe surface reaches very high values that entail higher interfacial potentials than those normally obtained. These extreme conditions encountered when the ES capillary is disconnected may lead to electrode reactions at the wire probe that would otherwise be inaccessible under normal conditions at the ES capillary walls. When the switch is made in the reverse direction-that is, when the ES capillary is reconnected and current production moves from the wire probe to the surrounding capillary-some transition time is observed and the wire probe momentarily becomes cathodic. This cathodic current balances the abruptly increased anodic current of the ES capillary without altering the total current. The consistency in current production is a clear manifestation of the controlledcurrent nature of the ES device.

Different potentials on wire electrode. In open-circuit potential measurements, such as those performed in obtaining DEEP maps, current is not allowed to flow through the wire probe. For current measurements, a zero-potential difference is set between the wire probe and ES capillary electrodes; thus, both share the job of current production. In negative-mode operation as in Figure $^{\circ} 8 \mathrm{a}^{\circ}{ }^{\circ}$ the ${ }^{\circ}$ predominant $^{\circ}$ electrode $^{\circ}$ reaction $^{\circ}$ is ${ }^{\circ} \mathrm{ca}^{-}$ thodic. The solution used consists of 1,4-benzoquinone, an ${ }^{\circ}$ asily ${ }^{\circ}$ reducible ${ }^{\circ}$ species $[36]^{\circ}{ }^{\circ} \mathrm{At}^{\circ} 2 \mathrm{mM}^{\circ}$ concentration, the reduction of benzoquinone is likely to provide most of the cathodic current. At $0.0 \mathrm{~V}$ difference between the wire probe and the ES capillary, the former contributes only a small fraction of the total current. If the wire potential is made negative $(-0.5 \mathrm{~V})$, it produces a substantially higher proportion of the cathodic current. However, even though the overall capability to generate reductive current is augmented, the total electrospray current does not change. Under these circumstances, the ES capillary is partially relieved of the burden of producing all the cathodic current and, in an

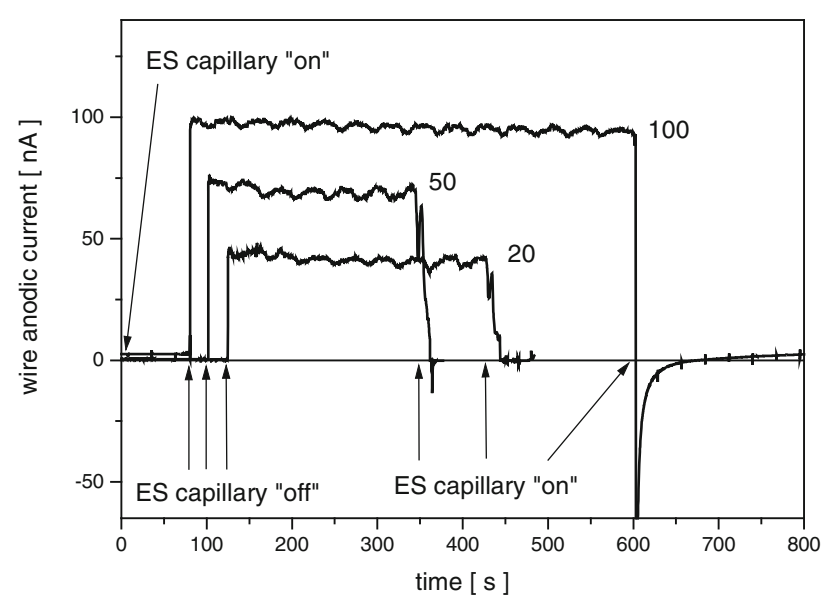

Figure 7. Changes of current to wire probe when ES capillary connection is switched on and off in the positive ion mode. The solution consists of $100 \%$ acetonitrile with added lithium trifluoromethylsulfonate supporting electrolyte at 20,50, and $100 \mu \mathrm{M}$. Note the rather long timescale of the experiment. 

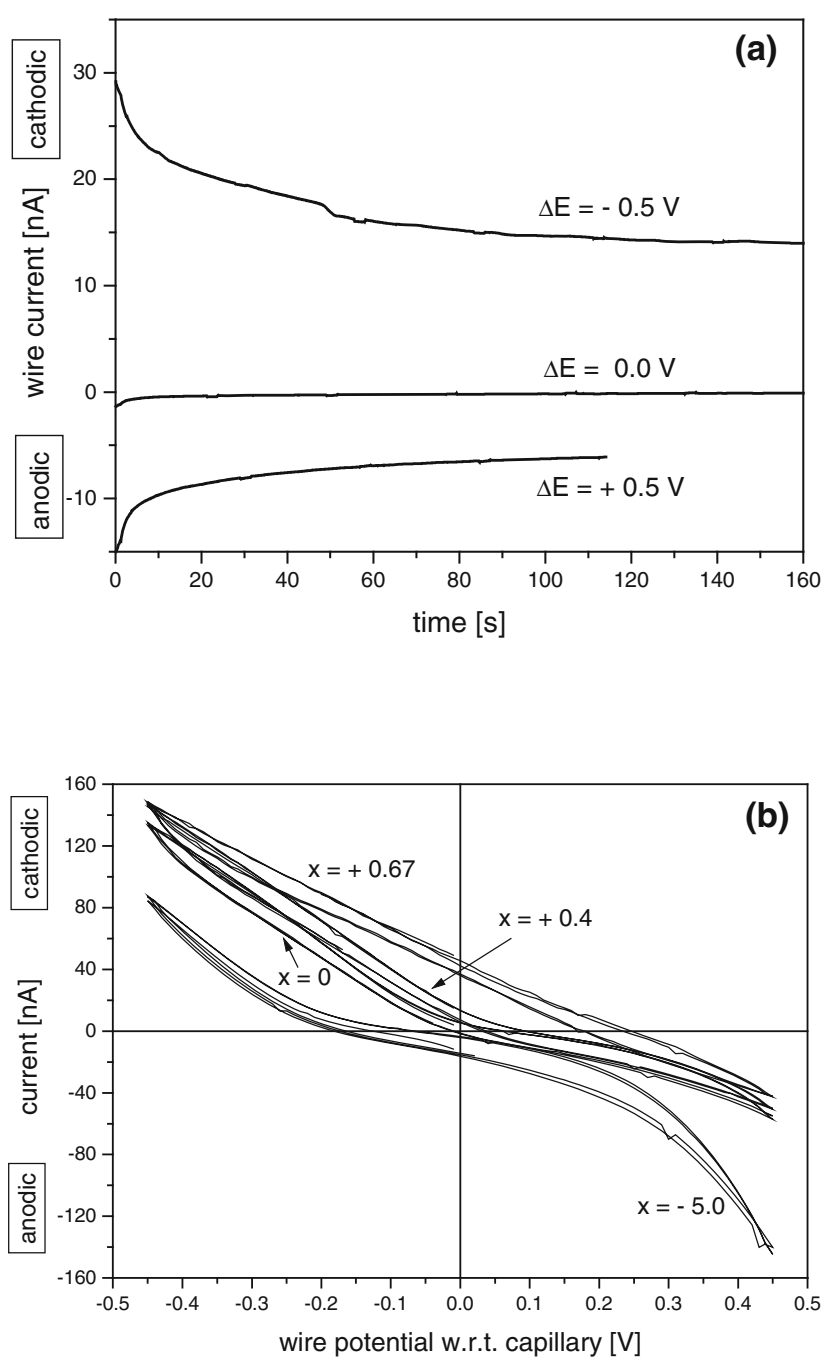

Figure 8. (a) Current to wire probe electrode as a function of time after start of negative ion mode electrospray operation when the potential difference between the wire probe and the ES capillary is set at the indicated value. The position of the wire probe is fixed at $x=-2.0 \mathrm{~mm}$. (b) Cyclic voltammetry in the ES capillary (negative ion mode) at a rate of $20 \mathrm{mV} / \mathrm{s}$. The total ES current is $110 \mathrm{nA}$ and the position of the wire probe electrode is designated in the plot. For both (a) and (b), the solution is $2000 \mu \mathrm{M}$ 1,4-benzoquinone with $190 \mu \mathrm{M}$ lithium trifluoromethylsulfonate supporting electrolyte in 1:1 ( $\mathrm{vol} / \mathrm{vol})$ methanol:chloroform.

extreme case of negative potential imposition at the wire probe, it could even produce an anodic current to maintain the total current at a constant cathodic value. If we now set the potential of the wire probe at a positive value $(+0.5 \mathrm{~V}$, lower curve), the wire probe will produce anodic (oxidative) current. To compensate for this, supplementary cathodic current must be produced by the ES capillary so that their sum remains constant (and overall cathodic). The shapes of the curves in Figure ${ }^{\circ} a^{\circ}$ will $^{\circ}$ change ${ }^{\circ}$ with ${ }^{\circ}$ probe ${ }^{\circ}$ position. ${ }^{\circ}$ Some ${ }^{\circ}$ oxidation products generated at the wire probe may be converted back to a reduced form at a downstream location of the ES capillary, although it is still quite possible to see anodic (oxidative) products in negative ion $\mathrm{ES}$ and vice versa.
Opposite currents produced at the separate electrodes not only act to balance the total current, but they also may serve to generate different electrochemical reaction products, and thus different mass spectra. This goal was previously accomplished with high conversion ratios by placing an electrochemical flow cell at an upstream ${ }^{\circ}$ position ${ }^{\circ}{ }^{\circ}{ }^{\circ} e^{\circ} \mathrm{ES}^{\circ} \mathrm{device} \odot[37,38]$. The ${ }^{\circ}$ benefit

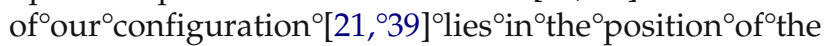
wire probe electrode, which can be placed inside the Taylor cone. The time needed for the electrolysis products to be transferred into the gas phase is thereby minimized; thus, electrochemical products are given less opportunity to undergo secondary reactions. A regular flow cell remains advantageous, however, when one wishes to electrolyze high concentrations of reactants by passing high currents.

Cyclic voltammetry. Polarization of the wire electrode with respect to the ES capillary can be done not only in a stepwise manner, but also in a continuous fashion, leading to a type of two-electrode cyclic voltammetry experiment. Cyclic voltammograms for four different positions ${ }^{\circ}$ of $^{\circ}$ the ${ }^{\circ}$ wire $^{\circ}$ probe $^{\circ}$ are $^{\circ}$ shown $^{\circ}$ in ${ }^{\circ}$ Figure $^{\circ} 8 \mathrm{~b} ;{ }^{\circ}$ a duplicate trace is shown for each scan to give an indication of reproducibility. During all data acquisition the total (cathodic) electrospray current remained constant at $110 \mathrm{nA}$, even when the wire probe cathodic current exceeded the total cathodic current (that is, the ES capillary was anodic) or when the wire probe was anodic. Notably, the shape, size, and position of the Taylor cone were not perceptibly altered during the voltage scans.

The $^{\circ}$ voltammograms ${ }^{\circ}$ in $^{\circ}$ Figure $^{\circ} 8 \mathrm{~b}^{\circ}$ appear ${ }^{\circ}$ to ${ }^{\circ} e^{\circ} \mathrm{com}-$ posites of conventional cyclic voltammograms to which a certain distortion function attributable to ES operation has been added. When the wire probe is placed deep within the capillary $(x=-5.0 \mathrm{~mm})$, the electrospray influence is practically negligible and the voltammogram looks similar to the conventional one. When the wire probe electrode is located at $x=0$, the central part of the voltammogram (where the potential difference between electrodes is close to zero) is no longer flat and the positive direction scan crosses the current axis at a cathodic value, indicating that current production extends beyond capacitative charging, in agreement with the above experiments. The uppermost voltammogram obtained for position $x=+0.67 \mathrm{~mm}$, where the wire probe is in contact with the skin of Taylor cone, is virtually a straight line. This type of linearity is characteristic of a voltammogram for two ideally nonpolarizable electrodes, showing primary current distribution features, where purely ohmic resistance is being measured. One can deduce from this voltammogram $(x=$ $+0.67 \mathrm{~mm}$ ) that at zero polarization the wire probe produces about $40 \%$ of the total ES cathodic current, whereas for a position $x=+0.40 \mathrm{~mm}$ it produces a meager $10 \%$. A sudden jump in current production can be explained by assuming that when the wire probe touches the skin of the Taylor cone it allows for a 
low-resistance contact to the ES capillary relative to pathways through the bulk electrolyte. The postulated separate conduction mechanisms through the bulk electrolyte and through the Taylor cone skin are thus corroborated. In the future, our wire probe may be able to obtain more quantitative data to determine what sets the competitive balance here. As previously mentioned, the skin is exposed directly to ambient gas, which further complicates the electrical conditions.

\section{Conclusions}

A good deal of effort has been expended to numerically and experimentally elucidate the effects of diffusion and flow on current distributions and concentration overpotentials in tubular flow electrochemical cells by the $\mathrm{o}^{\circ}$ Alkire ${ }^{\circ}\left[30,{ }^{\circ} 31\right]^{\circ}$ and $^{\circ}$ Bladel $^{\circ}[32]^{\circ}$ groups $^{\circ}$ However, primarily because of limiting assumptions, the specific numerical values that they obtained are not directly transferable to the situation in an electrospray capillary.

Another major difficulty in applying data from other studies to the ES device is the inconsistency with respect to the treatment of the stagnant layer and all accompanying consequences. Moreover, electrochemistry-ES studies have largely ignored turbulence of liquid flow inside the Taylor cone and theoretical treatments often use simplifying assumptions that render the final data only qualitatively useful.

The series of experiments presented here show that, because of its uniqueness, a description of the current distributions in the ES capillary requires a specific theoretical approach. However, we have also shown that the electrochemical environment in the electrospray capillary can be studied using conventional electrochemical methods and concepts. Current distributions have been measured by a method that, although somewhat crude, is still adequate to show essential features of electrospray source operation, some of which were unexpected on narrowly theoretical grounds. On the other hand, many features of the current maps were rooted in classical electrochemical concepts such as the fact that electrolytes of higher conductivity render the current distribution more uniform, whereas electrolytes of lower conductivity shift most of the current production to the region close to the capillary exit.

Close inspection of current maps reveals that these measurements present their own peculiarities arising from factors such as: mass transport limitations in the small region of active electrode surface, the narrow range of viable electrospray currents, and perturbations resulting from both ambient reactive gases and turbulent flow in the Taylor cone. Some current map findings do not correspond to features of potential maps, thus demonstrating the complementary nature of the two measurements and the value of performing both. For example, it has been shown that the possibilities for a variety of electrochemical processes, including those of contaminants, render current distributions subject to different regimes in various capillary regions such that the current map may or may not follow the expectations derived from the potential map. It has also been demonstrated that the self-regulating nature of electrospray, as a controlled-current electrochemical cell, makes ES operation resistant to production of extra currents of any polarity in the cell, even when the magnitude of such extraneous current exceeds the net ES current. Moreover, switching of current production from one place (one electrode) to another does not disturb the stability of electrospray operation.

The role of turbulence, Taylor cone "skin" conductivity, and the impact of ambient air were also demonstrated. The effects are substantive enough to throw into question an intuitive picture that considers only primary current features in the potential and current distributions.

Combining the present study with our previous investigations into potential mapping enables a more comprehensive view of the whole electrochemical process in the ES capillary. Further improvement of the understanding of the effects of electrochemistry and its specific consequences on mass spectra will come when the electrospray instrument is combined with a mass spectrometer, which allows for fast analysis of electrochemistry products, a step that is currently under way in our laboratory.

\section{Acknowledgments}

Financial support for this research was provided by National Science Foundation Grant CHE-0518288. The authors are also grateful to ESA Biosciences, Inc. for additional financial support for this project.

\section{References}

1. Bailey, A. G. Electrostatic Spraying of Liquids, John Wiley \& Sons: New York, 1988; pp 1-193.

2. Kebarle, P.; Ho, Y. On the Mechanism of Electrospray Mass Spectrometry. In Electrospray Ionization Mass Spectrometry, Cole, R. B., Ed.; John Wiley \& Sons: New York, 1997; pp 3-63.

3. Van Berkel, G. J. The Electrolytic Nature of Electrospray. In Electrospray Ionization Mass Spectrometry, Cole, R. B., Ed., John Wiley \& Sons: New York, 1997; pp 65-105.

4. Blades, A. T.; Ikonomou, M. G.; Kebarle, P. Mechanism of Electrospray Mass Spectrometry. Electrospray as an Electrolysis Cell. Anal. Chem. 1991, 63, 2109-2114.

5. Juhasz, P.; Ikonomou, M. G.; Blades, A. T.; Kebarle, P. Electrospray, Mechanism and Performance. In Methods and Mechanisms for Producing Ions from Large Molecules, Standing, K. G.; Ens, W., Eds.; Plenum Press: New York, 1991; pp 171-183.

6. de la Mora, J. F.; Van Berkel, G. J.; Enke, C. G.; Cole, R. B.; MartinezSanchez, M.; Fenn, J. B. Electrochemical Processes in Electrospray Ionization Mass Spectrometry. J. Mass Spectrom. 2000, 35, 939-952.

7. Cole, R. B. Some Tenets Pertaining to Electrospray Ionization Mass Spectrometry. J. Mass Spectrom. 2000, 35, 763-772.

8. Van Berkel, G. J.; Zhou, F. Characterization of an Electrospray Ion Source as a Controlled Current Electrolytic Cell. Anal. Chem. 1995, 67, 2916-2923.

9. Van Berkel, G. J.; Zhou, F. Electrospray as a Controlled-Current Electrolytic Cell: Electrochemical Ionization of Neutral Analytes for Detection by Electrospray Mass Spectrometry. Anal. Chem. 1995, 67, 3958tion by

10. Hager, D. B.; Dovichi, N. J. Behavior of Microscopic Liquid Droplets Near a Strong Electrostatic Field: Droplet Electrospray. Anal. Chem. 1994, 66, 1593-1594.

11. Li, Y.; Pozniak, B. P.; Cole, R. B. Mapping of Potential Gradients within the Electrospray Capillary. Anal. Chem. 2003, 75, 6987-6994.

12. Pozniak, B. P.; Cole, R. B. Negative Ion Mode Evolution of Potential Buildup and Mapping of Potential Gradients within the Electrospray Emitter. J. Am. Soc. Mass Spectrom. 2004, 15, 1737-1747. 
13. Bard, A. J.; Faulkner, L. R. Electrochemical Methods. Fundamentals and Applications. John Wiley \& Sons: New York, 1980.

14. Trasatti, S. The Electrode Potential. In Comprehensive Treatise of Electrochemistry, Vol. 1, Bockris, J. O’M.; Conway, B. E.; Yeager, E., Eds.; Plenum Press: New York, 1980; pp 45-82.

15. Levich, V. Physicochemical Hydrodynamics, Prentice Hall: Englewood Cliffs, NJ, 1962; pp 231-260.

16. Ibl, N. Fundamentals of Transport Phenomena in Electrolytic Systems. In Comprehensive Treatise of Electrochemistry, Vol. 6, Bockris, J. O'M.; Conway, B. E.; Yeager, E., Eds.; Plenum Press: New York, 1980; pp 1-63.

17. Ibl, N. Current Distribution. In Comprehensive Treatise of Electrochemistry, Vol. 6, Bockris, J. O'M.; Conway, B. E.; Yeager, E., Eds.; Plenum Press: New York, 1980; pp 239-315.

18. Newman, J. Transport Processes in Electrolytic Solutions. In Advances in Electrochemistry and Electrochemical Engineering, Vol. 5, Tobias, C. W., Ed.; Interscience Publishers, New York, 1967; pp 87-136.

19. West, A. C. Newman, J. Determination of Current Distributions Governed by Laplace's Equation. In Modern Aspects of Electrochemistry vol. 23, Conway, B. E.; Bockris, J. O'M.; White, R. E., Eds., Plenum Press, New York, 1992; pp 101-148.

20. Bockris, J. O'M.; Reddy, K. N. Modern Electrochemistry, Vol. 1, 2nd ed., Plenum Press: New York, 1998.

21. Xu, X.; Lu, W.; Cole, R. B. On-line Probe for Fast Electrochemistry/ Electrospray Mass Spectrometry. Investigation of Polycyclic Aromatic Hydrocarbons. Anal. Chem. 1996, 68, 4244-4253.

22. Clopeau, M.; Prunet-Foch, B. Electrohydrodynamic Spraying Functioning Modes: A Critical Review. J. Aerosol Sci. 1994, 25, 1021-1036.

23. de la Mora, J. F.; Loscertales, I. G. The Current Emitted by Highly Conducting Taylor Cones. J. Fluid Mech. 1994, 260, 155-184

24. Van Berkel, G. J.; Giles, G. E.; Bullock, J. S., IV; Gray, L. J. Computational Simulation of Redox Reactions within a Metal Electrospray Emitter. Anal. Chem. 1999, 71, 5288-5296.

25. Van Berkel, G. J. Electrolytic Deposition of Metals on to the High Voltage Contact in an Electrospray Emitter: Implications for Gas-phase Ion Formation. J. Mass Spectrom. 2000, 35, 773-783.

26. Hayati, I.; Bailey, A. I.; Tadros, T. F. Mechanism of Stable Jet Formation in Electrohydrodynamic Atomization. Nature 1986, 319, 41-43.

27. Hayati, I.; Bailey, A. I.; Tadros, T. F. Investigations into the Mechanisms of Electrohydrodynamic Spraying of Liquids. I. Effect of Electric Field and the Environment on Pendant Drops and Factors Affecting the
Formation of Stable Jets and Atomization. J. Colloid Interface Sci. 1987, 117, 205-221.

28. Hayati, I.; Bailey, A. I.; Tadros, T. F. Investigations into the Mechanisms of Electrohydrodynamic Spraying of Liquids. II. Mechanisms of Stable Jet Formation and Electrical Forces Acting on a Liquid Cone. J. Colloid Interface Sci. 1987, 117, 222-230.

29. Pozniak, B. P.; Cole, R. B. Ambient Gas Influence on Electrospray Potential as Revealed by DEEP Mapping Within the Electrospray Capillary, A 040716. Proceedings of the 52nd ASMS Conference on Mass Spectrometry and Allied Topics, Nashville, TN, May 2004.

30. Alkire, R.; Mirarefi, A. A. The Current Distribution Within Tubular Electrodes under Laminar Flow. J. Electrochem. Soc. 1973, 120, 1507-1515.

31. Alkire, R.; Mirarefi, A. A. Current Distribution in a Tubular Electrode under Laminar Flow: One Electrode Reaction. J. Electrochem. Soc. 1977, 124, 1043-1049.

32. Bladel, W. J.; Klatt, N. L. Reversible Charge Transfer at the Tubular Platinum Electrode. Anal. Chem. 1966, 38, 879-883.

33. Barrero, A.; Gañán-Calvo, A. M.; Davila, J.; Palacio, A.; GomezGonzalez, E. Low and High Reynolds Number Flows inside Taylor Cones. Phys. Rev. E 1998, 58, 7309-7314.

34. Tang, L.; Kebarle, P. Effect of the Conductivity of the Electrosprayed Solution on the Electrosprayed Current. Factors Determining Analyte Sensitivity in Electrospray Mass Spectrometry. Anal. Chem. 1991, 63, 2709-2715.

35. Dibble, T.; Bandyopadhyay, S.; Ghoroghchian, J.; Smith, J. J.; Sarfarazi, F.; Fleischmann, M.; Pons, S. Electrochemistry at Very High Potentials: Oxidation of the Rare Gases and Other Gases in Nonaqueous Solvents at Ultramicroelectrodes. J. Phys. Chem. 1986, 90, 5275-5277.

36. Popp, F.; Schultz, H. P. Electrolytic Reduction of Organic Compounds. Chem. Rev. 1961, 61, 19-40.

37. Van Berkel, G. J.; Asano, G. K.; Granger, M. C. Controlling Analyte Electrochemistry in an Electrospray Ion Source with a Three Electrode Emitter Cell. Anal. Chem. 2004, 76, 1493-1499.

38. Van Berkel, G. J.; Kertesz, V.; Ford, M. J.; Granger, M. C. Efficient Analyte Oxidation in an Electrospray Ion Source Using a Porous Flow-Through Electrode Emitter. J. Am. Soc. Mass Spectrom. 2004, 15, 1755-1766.

39. Lu, W.; Xu, X.; Cole, R. B. On-Line Linear Sweep VoltammetryElectrospray Mass Spectrometry. Anal. Chem. 1997, 69, 2478-2484. 